\title{
Implicit factorization of unbalanced RSA moduli
}

\author{
Abderrahmane Nitaj ${ }^{1}$ and Muhammad Rezal Kamel Ariffin ${ }^{2}$ \\ 1 Laboratoire de Mathématiques Nicolas Oresme \\ Université de Caen Basse Normandie, France \\ abderrahmane.nitaj@unicaen.fr \\ 2 Al-Kindi Cryptography Research Laboratory, \\ Institute for Mathematical Research, \\ Universiti Putra Malaysia (UPM), Selangor, Malaysia \\ rezal@upm.edu.my
}

\begin{abstract}
Let $N_{1}=p_{1} q_{1}$ and $N_{2}=p_{2} q_{2}$ be two RSA moduli, not necessarily of the same bit-size. In 2009, May and Ritzenhofen proposed a method to factor $N_{1}$ and $N_{2}$ given the implicit information that $p_{1}$ and $p_{2}$ share an amount of least significant bits. In this paper, we propose a generalization of their attack as follows: suppose that some unknown multiples $a_{1} p_{1}$ and $a_{2} p_{2}$ of the prime factors $p_{1}$ and $p_{2}$ share an amount of their Most Significant Bits (MSBs) or an amount of their Least Significant Bits (LSBs). Using a method based on the continued fraction algorithm, we propose a method that leads to the factorization of $N_{1}$ and $N_{2}$. Using simultaneous diophantine approximations and lattice reduction, we extend the method to factor $k \geq 3 \mathrm{RSA}$ moduli $N_{i}=p_{i} q_{i}$ $i=1, \ldots, k$ given the implicit information that there exist unknown multiples $a_{1} p_{1}, \ldots, a_{k} p_{k}$ sharing an amount of their MSBs or their LSBs. Also, this paper extends many previous works where similar results were obtained when the $p_{i}$ 's share their MSBs or their LSBs.
\end{abstract}

\section{Introduction}

Research in determining pre-requisites for strong primes for the integer factorization problem (IFP) of a product of two primes $N=p q$ has been intriguing and have captured the attention of researchers since IFP came into prominence via the RSA algorithm. The simplicity of the problem statement raised interest on whether such a simple problem statement describing the IFP could only be solved in exponential time for all cases, i.e. all types of primes. As can be found in the literature, this is not the case. So-called weak primes were identified by researchers and this caused an avalanche of research output on this matter. In this paper, we focus on IFP when $N=p q$ is unbalanced, that is when $q$ is much smaller than $p$.

In PKC 2009, May and Ritzenhofen [5] presented a method for factoring large integers with some implicit hints. More precisely, let $N_{1}=p_{1} q_{1}$ and $N_{2}=p_{2} q_{2}$ be two RSA moduli of the same bit-size such that $q_{1}$ and $q_{2}$ are $\alpha$-bit primes and $p_{1}$ and $p_{2}$ share at least $t$ least significant bits (LSBs). The method of May and Ritzenhofen is a lattice based method that allows to find the factorization 
of $N_{1}$ and $N_{2}$ when $t \geq 2 \alpha+3$. May and Ritzenhofen's method heuristically generalizes to a lattice based method to simultaneously factor $k$ RSA moduli $N_{1}=p_{1} q_{1}, \ldots, N_{k}=p_{k} q_{k}$ when the $p_{i}$ 's share $t \geq \frac{k}{k-1} \alpha$ many LSBs.

In [8], Sarkar and Maitra reconsidered the method of May and Ritzenhofen for two RSA moduli. Sarkar and Maitra's method works when $N_{1}=p_{1} q_{1}$ and $N_{2}=p_{2} q_{2}$ are such that $p_{1}$ and $p_{2}$ share their LSBs or most significant bits (MSBs) as well as a contiguous portion of bits at the middle.

In PKC 2010, Faugère, Marinier and Renault [1] presented a new and rigorous lattice-based method that addresses the implicit factoring problem when $p_{1}$ and $p_{2}$ share $t$ MSBs. Moreover, when $N_{1}=p_{1} q_{1}$ and $N_{2}=p_{2} q_{2}$ are two RSA moduli of the same bit-size and the prime factors $q_{i}$ are $\alpha$-bit primes, the method of Faugère et al. provably factors $N_{1}$ and $N_{2}$ as soon as $p_{1}$ and $p_{2}$ share $t \geq 2 \alpha+3$ MSBs. The method heuristically generalizes to the case when $p_{1}$ and $p_{2}$ share an amount of bits in the middle. It also heuristically generalizes to $k$ RSA moduli $N_{1}=p_{1} q_{1}, \ldots, N_{k}=p_{k} q_{k}$ when the $p_{i}$ 's share $t \geq \frac{k}{k-1} \alpha+6$ of MSBs.

In IWSEC 2013, Kurosawa and Ueda 3 presented a lattice-based method to factor two RSA moduli $N_{1}=p_{1} q_{1}$ and $N_{2}=p_{2} q_{2}$ of the same bit size when $p_{1}$ and $p_{2}$ share $t$ LSBs with $t \geq 2 \alpha+1$ where $q_{1} \approx q_{2} \approx 2^{\alpha}$. Their method takes advantage on using Gaussian reduction techniques. It slightly improves the bound $t \geq 2 \alpha+3$ of May and Ritzenhofen. We notice that Kurosawa and Ueda did not study a number of possible extensions of their method, namely, when $p_{1}$ and $p_{2}$ share $t$ MSBs and also when the multiple of the primes share LSB's and MSB's.

All the former attacks apply when the RSA moduli $N_{1}=p_{1} q_{1}, \ldots, N_{k}=p_{k} q_{k}$ are of the same bit-size and the $p_{i}$ 's share an amount of MSBs, LSBs or bits in the middle. In this paper, we present novel approaches of implicit factoring that generalize the former attacks and apply when some unknown multiples $a_{i} p_{i}$ of the prime factors $p_{i}$ share an amount of MSBs or of LSBs.

Our first method concerns two RSA moduli $N_{1}=p_{1} q_{1}, N_{2}=p_{2} q_{2}$ of arbitrarily sizes in the situation that there exist two integers $a_{1}, a_{2}$ such that $a_{1} p_{1}$ and $a_{2} p_{2}$ share $t$ many MSBs. We show that, using the continued fraction expansion of $\frac{N_{2}}{N_{1}}$, one can factor simultaneously $N_{1}$ and $N_{2}$ whenever $\left|a_{1} p_{1}-a_{2} p_{2}\right|<\frac{p_{1}}{2 a_{2} q_{1} q_{2}}$. In particular, when $N_{1}$ and $N_{2}$ are of the same bit size and $q_{1}, q_{2}$ are $\alpha$-bit primes, then one can factor $N_{1}$ and $N_{2}$ whenever $a_{i} \leq 2^{\beta}$ for $i=1,2$ and $t \geq 2 \alpha+2 \beta+1$. When $\beta=0$, that is $a_{1}=a_{2}=1$, our result becomes $t \geq 2 \alpha+1$ and improves the bound $t \geq 2 \alpha+3$ presented in [8] and [1] where the methods are based on lattice reduction techniques.

Our second method is a heuristic generalization of the first method to an arbitrary number $k \geq 3$ of RSA moduli $N_{i}=p_{i} q_{i}, i=1, \ldots, k$ in the situation that there exist $k$ integers $a_{i}$ such that the $a_{i} p_{i}$ 's share $t$ many MSBs. When the RSA moduli are of the same bit size and the factors $q_{i}, i=1, \ldots, k$, are $\alpha$-bit primes, the method allows us to factor the RSA moduli as soon as

$$
t>\frac{k}{k-1} \alpha+\frac{k^{2}}{k-1} \beta+\frac{k}{2(k-1)}\left(1+\log _{2}(\pi e)\right)
$$


where $\beta$ is such that $a_{i} \leq 2^{\beta}$. Once again, with $\beta=0$, we improve the bound presented in the attack of [1].

Our third method addresses the implicit factoring problem when two unbalanced RSA moduli $N_{1}=p_{1} q_{1}$ and $N_{2}=p_{2} q_{2}$ of arbitrarily sizes are such that there exist two integers $a_{1}$ and $a_{2}$ such that $a_{1} p_{1}$ and $a_{2} p_{2}$ share $t$ many LSBs. We show that it is possible to factor both $N_{1}$ and $N_{2}$ if $a_{1} a_{2} q_{1} q_{2}<2^{t-1}$. This method is also based on the continued fraction algorithm, applied to $\frac{T}{2^{t}}$ where $T \equiv N_{2} N_{1}^{-1}\left(\bmod 2^{t}\right)$. We notice that, when $a_{1}=a_{2}=1$ and $q_{1}, q_{2}$ are $\alpha$-bit primes, the former condition on $t$ transforms to $t \geq 2 \alpha+1$ which improves the bound on $t$ for LSBs in [5] and [8] and retrieves the bound of [3].

Our fourth method is a generalization of the third method to $k \geq 3 \mathrm{RSA}$ moduli $N_{i}=p_{i} q_{i}, i=1, \ldots, k$. Assume that there exist $k$ integers $a_{i}$ such that the $a_{i} p_{i}$ 's share $t$ many LSBs. If the RSA moduli are of the same bit size and the $q_{i}$ 's are $\alpha$-bit primes, our method allows us to address the implicit factoring problem whenever $t$ satisfies (1) where $\beta$ is such that $a_{i} \leq 2^{\beta}$.

In fact our findings under the four scenarios, further discus possible malicious key generation of RSA moduli by observing not only the difference between primes, but also the differences of the multiple of primes. At the same time it generalizes the previous works by [5], [8], [1] and [3]. Contrarily to the previous works, we study all the possible situations involving $k=2$ as well as $k \geq 3$ in both cases of MSBs and LSBs. In Table 1, we compare the applicability of our methods against the previous methods for the different scenarios.

Table 1. Applicability of the methods for $k$ RSA moduli.

\begin{tabular}{||l|c|c|c|c||}
\hline Method & \multicolumn{2}{|c|}{ MSBs } & \multicolumn{2}{c|}{ LSBs } \\
\hline & $k=2$ & $k \geq 3$ & $k=2$ & $k \geq 3$ \\
\hline May, Ritzenhofen [5] & No & No & Yes & Yes \\
\hline Sarkar, Maitra [8] & Yes & No & Yes & No \\
\hline Faugère et al. [1] & Yes & Yes & No & No \\
\hline Kurosawa, Ueda [3] & No & No & Yes & No \\
\hline Our methods & Yes & Yes & Yes & Yes \\
\hline
\end{tabular}

Also, we notice that not only the new bounds improve the previous ones, but also that the rank of the new underlying lattices are often lower than the ranks of the lattices used in the former methods. In Table 2 and Table 3 , we compare our results against the former results with $k$ RSA moduli in terms of bounds and dimension of the lattices.

We apply our results to the implicit factorization of $k \geq 2$ RSA for Paranoids [7] $N_{i}=p_{i} q_{i}, i=1, \ldots, k$, where $p_{i} \approx 2^{4500}$ and $q_{i} \approx 2^{500}$. For example, we show that we can easily factor two RSA for Paranoids moduli $N_{1}=p_{1} q_{1}$, $N_{2}=p_{2} q_{2}$ if there exist two integers $a_{1}$ and $a_{2}$ such that $a_{1} p_{1}$ and $a_{2} p_{2}$ share $t$ MSBs or $t$ LSBs with $t \geq 1001+2 \beta$ where $\beta$ is such that $a_{i} \leq 2^{\beta}$ for $i=1,2$. 
Table 2. Comparison of the bounds on $t$ for $k$ RSA moduli in the MSB case.

\begin{tabular}{|c|c|c|}
\hline Method for MSBs & $\begin{array}{l}\text { Number of RSA moduli } \\
k=2\end{array}$ & Number of RSA moduli $k \geq 3$ \\
\hline May, Ritzenhofen [5] & Not studied & Not studied \\
\hline Sarkar, Maitra 8] & $\begin{array}{l}\text { For } q_{1} \approx q_{2} \approx 2^{\alpha} \\
\text { and }\left|p_{1}-p_{2}\right|<2^{t}, \\
\text { the bound is heuristi- } \\
\text { cally better than } t \geq \\
2 \alpha+3 \text { and the dimension } \\
\text { of the lattice is at least } 9 \\
(m=t=1) .\end{array}$ & Can not be applied \\
\hline Faugère et al. [1] & $\begin{array}{l}\text { For } q_{1} \approx q_{2} \approx 2^{\alpha} \text { and } \\
\left|p_{1}-p_{2}\right|<2^{t}, \text { the rigor- } \\
\text { ous bound is } t \geq 2 \alpha+3 \\
\text { using 2-dimensional lat- } \\
\text { tices of } \mathbb{Z}^{3} \text {. }\end{array}$ & $\begin{array}{l}\text { For } q_{1} \approx \ldots \approx q_{k} \approx 2^{\alpha} \text { and } \\
\left|p_{i}-p_{j}\right|<2^{t}, \text { the heuristic } \\
\text { bound is } t>\frac{k}{k-1} \alpha+1+ \\
\frac{k}{2(k-1)}\left(2+\frac{\log _{2}(k)}{2}+\log _{2}(\pi e)\right) \\
\text { using } k \text {-dimensional lattices of } \\
\mathbb{Z}^{\frac{k(k+1)}{2}} \text {. }\end{array}$ \\
\hline Kurosawa, Ueda 3] & Not studied. & Can not be applied \\
\hline Our results & $\begin{array}{l}\text { For } q_{1} \approx q_{2} \approx 2^{\alpha} \text { and } \\
\left|a_{1} p_{1}-a_{2} p_{2}\right|<2^{t} \text { for } \\
\text { some unknown integers } \\
a_{1}, a_{2} \leq 2^{\beta}, \text { the rigorous } \\
\text { bound is } t \geq 2 \alpha+2 \beta+1 \\
\text { using the continued frac- } \\
\text { tion algorithm. For } a_{1}= \\
a_{2}=1, \beta=0 \text { and the } \\
\text { the rigorous bound is } t \geq \\
2 \alpha+1\end{array}$ & $\begin{array}{l}\text { For } q_{1} \approx \ldots \approx q_{k} \approx 2^{\alpha} \text { and } \\
\left|a_{i} p_{i}-a_{j} p_{j}\right|<2^{t} \text { for some un- } \\
\text { known integers } a_{1}, \ldots, a_{k} \text {, the } \\
\text { heuristic bound is } t>\frac{k}{k-1} \alpha+ \\
\frac{k^{2}}{k-1} \beta+\frac{k}{2(k-1)}\left(1+\log _{2}(\pi e)\right) \text { us- } \\
\text { ing } k \text {-dimensional lattices of } \mathbb{Z}^{k} \text {. } \\
\text { For } a_{1}=\ldots=a_{k}=1 \text {, } \\
\beta=0 \text { and the the heuris- } \\
\text { tic bound is } t>\frac{k}{k-1} \alpha+ \\
\frac{k}{2(k-1)}\left(1+\log _{2}(\pi e)\right) \text {. }\end{array}$ \\
\hline
\end{tabular}


Table 3. Comparison of the bounds on $t$ for $k$ RSA moduli in the LSB case.

\begin{tabular}{|c|c|c|}
\hline Method for LSBs & $\begin{array}{l}\text { Number of RSA moduli } \\
k=2\end{array}$ & Number of RSA moduli $k \geq 3$ \\
\hline May, Ritzenhofen [5] & $\begin{array}{l}\text { For } q_{1} \approx q_{2} \approx 2^{\alpha} \\
\text { and } p_{1} \equiv p_{2}\left(\bmod 2^{t}\right) \\
\text { the rigorous bound is } \\
t \geq 2 \alpha+3 \text { using } 2- \\
\text { dimensional lattices of } \\
\mathbb{Z}^{2} \text {. }\end{array}$ & $\begin{array}{l}\text { For } q_{1} \approx \ldots \approx q_{k} \approx 2^{\alpha} \text { and } \\
p_{i} \equiv p_{j}\left(\bmod 2^{t}\right), \text { the heuris- } \\
\text { tic bound is } t \geq \frac{k}{k-1} \alpha \text { using } k \text { - } \\
\text { dimensional lattices of } \mathbb{Z}^{k} \text {. }\end{array}$ \\
\hline Sarkar, Maitra 8] & $\begin{array}{l}\text { For } q_{1} \approx q_{2} \approx 2^{\alpha} \\
\text { and } p_{1} \equiv p_{2}\left(\bmod 2^{t}\right), \\
\text { the bound is heuristi- } \\
\text { cally better than } t \geq \\
2 \alpha+3 \text { and the dimension } \\
\text { of the lattice is at least } 9 \\
(m=t=1) .\end{array}$ & Can not be applied. \\
\hline Faugère et al. 1] & Not studied. & Not studied. \\
\hline Kurosawa, Ueda 3 & $\begin{array}{l}\text { For } q_{1} \approx q_{2} \approx 2^{\alpha} \\
\text { and } p_{1} \equiv p_{2}\left(\bmod 2^{t}\right) \\
\text { the rigorous bound is } \\
t \geq 3 \alpha+1 \text { using } 2- \\
\text { dimensional lattices of } \\
\mathbb{Z}^{2} \text {. }\end{array}$ & Can not be applied \\
\hline Our results & $\begin{array}{l}\text { For } q_{1} \approx q_{2} \approx 2^{\alpha} \text { and } \\
\left|a_{1} p_{1}-a_{2} p_{2}\right|<2^{t} \text { for } \\
\text { some unknown integers } \\
a_{1}, a_{2} \leq 2^{\beta}, \text { the rigorous } \\
\text { bound is } t \geq 2 \alpha+2 \beta+1 \\
\text { using the continued frac- } \\
\text { tion algorithm. For } a_{1}= \\
a_{2}=1, \beta=0 \text { and the } \\
\text { the rigorous bound is } t \geq \\
2 \alpha+1 .\end{array}$ & $\begin{array}{l}\text { For } q_{1} \approx \ldots \approx q_{k} \approx \\
2^{\alpha} \text { and } a_{i} p_{i} \equiv a_{j} p_{j}\left(\bmod 2^{t}\right) \\
\text { for some unknown integers } \\
a_{1}, \ldots, a_{k}, \text { the heuristic bound } \\
\text { is } t>\frac{k}{k-1} \alpha+\frac{k^{2}}{k-1} \beta+ \\
\frac{k}{2(k-1)}\left(1+\log _{2}(\pi e)\right) \text { using } k- \\
\text { dimensional lattices of } \mathbb{Z}^{k} \text {. For } \\
a_{1}=\ldots=a_{k}=1, \beta=0 \text { and } \\
\text { the the heuristic bound is } t> \\
\frac{k}{k-1} \alpha+\frac{k}{2(k-1)}\left(1+\log _{2}(\pi e)\right) .\end{array}$ \\
\hline
\end{tabular}


The rest of this paper is organized as follows. In Section 2, we introduce some useful background on continued fractions and lattice basis reduction. In section 3 , we present our first method to address the problem of implicit factoring of two RSA moduli $N_{1}=p_{1} q_{1}$ and $N_{2}=p_{2} q_{2}$ when $a_{1} p_{1}$ and $a_{2} p_{2}$ share $t$ MSBs. In section 4 , we present a generalization to $k \geq 3 \mathrm{RSA}$ moduli $N_{i}=p_{i} q_{i}$, $i=1, \ldots, k$, in the situation that the $a_{i} p_{i}$ 's share $t$ MSBs. In section 5 , we present an attack on two RSA moduli $N_{1}=p_{1} q_{1}$ and $N_{2}=p_{2} q_{2}$ when $a_{1} p_{1}$ and $a_{2} p_{2}$ share $t$ LSBs and we generalize this attack to $k \geq 3$ RSA moduli in Section 6. In Section 7, we present our experiments and we conclude in Section 8.

\section{Preliminaries}

In this section, we review some knowledge background on continued fractions and lattice basis reduction.

\subsection{Continued fractions}

First we give the definition of continued fractions and state a related theorem. The details can be referenced in [2]. For any positive real number $\xi$, define $\xi_{0}=\xi$ and for $i=0,1, \ldots, n, a_{i}=\left\lfloor\xi_{i}\right\rfloor, \xi_{i+1}=1 /\left(\xi_{i}-a_{i}\right)$ unless $\xi_{n}$ is an integer. Then $\xi$ can be expanded as a continued fraction in the following form

$$
x=a_{0}+\frac{1}{a_{1}+\frac{1}{\ddots+\frac{1}{a_{n}+\frac{1}{\ddots}}}},
$$

which, for simplicity, can be rewritten as $\xi=\left[a_{0}, a_{1}, \ldots, a_{n}, \ldots\right]$. If $\xi$ is a rational number, then the process of calculating the continued fraction expansion would be finished in some finite index $n$ and then $\xi=\left[a_{0}, a_{1}, \ldots, a_{n}\right]$. The convergents $\frac{a}{b}$ of $\xi$ are the fractions defined by $\frac{a}{b}=\left[a_{0}, \ldots, a_{i}\right]$ for $i \geq 0$. We note that, if $\xi=\frac{a}{b}$ is a rational number, then the continued fraction expansion of $\xi$ is finite with the total number of convergents being polynomial in $\log (b)$.

Another important result on continued fractions that will be used throughout this paper is the following (Theorem 184 of [2]).

Theorem 1 (Legendre). Let $\xi$ be a positive number. Suppose $\operatorname{gcd}(a, b)=1$ and

$$
\left|\xi-\frac{a}{b}\right|<\frac{1}{2 b^{2}}
$$

Then $\frac{a}{b}$ is one of the convergents of the continued fraction expansion of $\xi$. 


\subsection{Lattice reduction}

Let us present some basics on lattice reduction techniques. Let $b_{1} \ldots, b_{d}$ be $d$ linearly independent vectors of $\mathbb{R}^{n}$ with $d \leq n$. The set of all integer linear combinations of the $b_{i}$ forms a lattice $\mathcal{L}$. Namely,

$$
\mathcal{L}=\left\{\sum_{i=1}^{d} x_{i} b_{i} \mid x_{i} \in \mathbb{Z}\right\} .
$$

The integer $n$ is the rank of the lattice $\mathcal{L}$ and $d$ is its dimension. The set $\left(b_{1}, \ldots, b_{d}\right)$ is called a basis of $\mathcal{L}$. The determinant of $\mathcal{L}$ is $\operatorname{defined}$ as $\operatorname{det}(\mathcal{L})=$ $\sqrt{B^{t} B}$ where $B$ is the basis matrix, i.e., the matrix of the $b_{i}$ 's in the canonical basis of $\mathbb{R}^{n}$. The determinant is invariant under unimodular basis transformations of $B$ and reduces to $\operatorname{det}(\mathcal{L})=|\operatorname{det}(B)|$ when $d=n$. Let us denote by $\|v\|$ the Euclidean norm of a vector $v \in \mathcal{L}$. A central problem in lattice reduction is to find short non-zero vectors in $\mathcal{L}$. Vectors with short norm can be computed by the LLL algorithm of Lenstra, Lenstra, and Lovász 4 .

Theorem 2 (LLL). Let $\mathcal{L}$ be a lattice spanned by a basis $\left(u_{1}, \ldots, u_{d}\right)$. Then the $L L L$ algorithm produces a new basis $\left(b_{1}, \ldots, b_{d}\right)$ of $\mathcal{L}$ satisfying

$$
\left\|b_{1}\right\| \leq 2^{\frac{d-1}{4}} \operatorname{det}(\mathcal{L})^{\frac{1}{d}} .
$$

On the other hand, for comparison, the Gaussian Heuristic says that the length of the shortest non-zero vector of a lattice $\mathcal{L}$ is usually approximately $\sigma(\mathcal{L})$ where

$$
\sigma(\mathcal{L}) \approx \sqrt{\frac{d}{2 \pi e}} \operatorname{det}(\mathcal{L})^{\frac{1}{d}}
$$

\section{Factoring two RSA Moduli in the MSB Case}

In this section, we study the problem of factoring two RSA moduli $N_{1}=p_{1} q_{1}$ and $N_{2}=p_{2} q_{2}$ where $a_{1} p_{1}$ and $a_{2} p_{2}$ coincide on the $t$ most significant bits (MSBs), that is when $\left|a_{2} p_{2}-a_{1} p_{1}\right|$ is sufficiently small.

\subsection{The general attack for two RSA Moduli in the MSB Case}

We begin by the following result which applies to two RSA moduli not necessarily of the same bit size.

Theorem 3. Let $N_{1}=p_{1} q_{1}, N_{2}=p_{2} q_{2}$ be two RSA moduli. If there exist two integers $a_{1}, a_{2}$ such that $a_{1}<p_{2}, a_{2}<p_{1}$ and $\left|a_{1} p_{1}-a_{2} p_{2}\right|<\frac{p_{1}}{2 a_{2} q_{1} q_{2}}$, then one can factor $N_{1}$ and $N_{2}$ in polynomial time.

Proof. For $N_{1}=p_{1} q_{1}$ and $N_{2}=p_{2} q_{2}$, let $x=a_{1} p_{1}-a_{2} p_{2}$. Multiplying $x$ by $q_{2}$, we get $a_{1} p_{1} q_{2}-a_{2} N_{2}=x q_{2}$. Suppose that $|x|<\frac{p_{1}}{2 a_{2} q_{1} q_{2}}$. Then, dividing by $a_{2} N_{1}=a_{2} p_{1} q_{1}$, we get

$$
\left|\frac{N_{2}}{N_{1}}-\frac{a_{1} q_{2}}{a_{2} q_{1}}\right|=\frac{|x| q_{2}}{a_{2} p_{1} q_{1}}<\frac{p_{1}}{2 a_{2} q_{1} q_{2}} \times \frac{q_{2}}{a_{2} p_{1} q_{1}}=\frac{1}{2\left(a_{2} q_{1}\right)^{2}} .
$$


Hence, from Theorem 1 it follows that $\frac{a_{1} q_{2}}{a_{2} q_{1}}$, in lowest term is one of the convergents in the continued fraction expansion of $\frac{N_{2}}{N_{1}}$. If we assume $a_{1}<p_{2}, a_{2}<p_{1}$, then using $\frac{a_{1} q_{2}}{a_{2} q_{1}}$, we get $q_{1}=\operatorname{gcd}\left(N_{1}, a_{2} q_{1}\right)$ and therefore $p_{1}=\frac{N_{1}}{q_{1}}$. Similarly, we get $q_{1}=\operatorname{gcd}\left(N_{2}, a_{1} q_{2}\right)$ and $p_{2}=\frac{N_{2}}{q_{2}}$.

Remark 1. The result of Theorem 3 is valid even when the RSA moduli are not of the same size. Comparatively, the attacks presented by Sarkar and Maitra in [8] and Faugère et al. in [1] are valid only if $N_{1} \approx N_{2}$ and $q_{1} \approx q_{2}$.

Example 1. Consider the following RSA moduli

$$
\begin{aligned}
& N_{1}=63431782986412625310912155582547071972279848634479, \\
& N_{2}=9946006657067710178027582903059286609914354223 .
\end{aligned}
$$

The first partial quotients of $\frac{N_{2}}{N_{1}}$ are

$$
\begin{aligned}
& {[0,6377,1,1,1,1,2,2,3,1,1,3,9,1,1,1,1,7,1,19,1,1,11,} \\
& 1,1,23,1,1,3,2,3,2,3,4,2,1,1,1,8,1,322,3,4,1,1,2, \ldots]
\end{aligned}
$$

Each convergent $\frac{a}{b}$ of $\frac{N_{2}}{N_{1}}$ is a candidate for $\frac{a_{1} q_{2}}{a_{2} q_{1}}$ and the good one will reveal $q_{1}$ and $q_{2}$ if the conditions of Theorem 3 are fulfilled. Indeed, the 40th convergent is $\frac{a}{b}=\frac{1351300027964332}{8618068847003717463}$ and gives

$$
\begin{aligned}
& q_{1}=\operatorname{gcd}\left(N_{1}, b\right)=2125300178867 \\
& p_{1}=\frac{N_{1}}{q_{1}}=29846034747067203786403150576377329237 \\
& q_{2}=\operatorname{gcd}\left(N_{2}, a\right)=9531501481 \\
& p_{2}=\frac{N_{2}}{q_{2}}=1043487920228935667940393294165327383 .
\end{aligned}
$$

We notice that $p_{1}$ and $p_{2}$ do not share any amount of LSBst nor MSBs nor bits in the middle. This shows that the attacks presented in [8] and [1] will not give a result in this situation.

\subsection{Application to unbalanced RSA and RSA for Paranoids}

As an application of Theorem 3 to factor two unbalanced RSA moduli of the same bit-size, we get the following result.

Corollary 1. Let $N_{1}=p_{1} q_{1}, N_{2}=p_{2} q_{2}$ be two unbalanced RSA moduli of the same bit-size $n$. Suppose that $q_{i} \approx 2^{\alpha}, p_{i} \approx 2^{n-\alpha}$ for $i=1,2$. Let $a_{1}, a_{2}$ be two integers such that $a_{i} \leq 2^{\beta}, i=1,2$. If $a_{1} p_{1}$ and $a_{2} p_{2}$ share $t$ most significant bits with $t \geq 2 \alpha+2 \beta+1$, then one can factor $N_{1}$ and $N_{2}$ in polynomial time.

Proof. Let $N_{1}=p_{1} q_{1}, N_{2}=p_{2} q_{2}$ be two RSA moduli with $N_{1} \approx N_{2} \approx 2^{n}$ and $q_{1} \approx q_{2} \approx 2^{\alpha}$. Suppose that a multiple $a_{1} p_{1}$ and a multiple $a_{2} p_{2}$ share the $t$ 
most significant bits, that is $a_{1} p_{1}-a_{2} p_{2}=x$ with $|x| \leq 2^{n-\alpha+\beta-t}$. Assume that $t \geq 2 \alpha+2 \beta+1$. Then

$$
2 a_{2} q_{1} q_{2}|x|<2^{1+\beta+2 \alpha+n-\alpha+\beta-t} \leq 2^{n-\alpha} \approx p_{1},
$$

which can be transformed into the inequality $|x|<\frac{p_{1}}{2 a_{2} q_{1} q_{2}}$. Hence, as in Theorem 3 it follows that $\frac{a_{1} q_{2}}{a_{2} q_{1}}$ is a convergent of the continued fraction of $\frac{N_{2}}{N_{1}}$ which leads to the factorization of $N_{1}$ and $N_{2}$.

Remark 2. If we consider $\beta=0$ in Corollary 1 that is, if $a_{1}=a_{2}=1$, a sufficient condition to factor the two RSA moduli is $t \geq 2 \alpha+1$ which slightly improves the bound $t \geq 2 \alpha+3$ found by Faugère et al. in [1]. This shows that the bound found by Faugère et al. with lattice reduction techniques can be achieved using the continued fraction algorithm instead.

Consider two RSA for Paranoids moduli $N_{i}=p_{i} q_{i}$ with $N_{i} \approx 2^{5000}, q_{i} \approx 2^{500}$ and $p_{i} \approx 2^{4500}$ for $i=1,2$. Then $\alpha=500$ and by Corollary 1 it is possible to factor $N_{1}$ and $N_{2}$ if a multiple $a_{1} p_{1}$ and a multiple $a_{2} p_{2}$ share the $t$ MSBs whenever $t \geq 2 \alpha+2 \beta+1$, that is whenever $t \geq 1001+2 \beta$.

\section{Factoring $k$ RSA Moduli in the MSB Case}

The attack mounted for two RSA moduli can be generalized to an arbitrary number $k \geq 3$ of moduli $N_{i}=p_{i} q_{i}, i=1 \ldots, k$ where the $q_{i}$ 's are $\alpha$-bit primes and the $a_{i} p_{i}$ 's share $t$ MSBs. Instead of using the continued fraction algorithm, we use a lattice based method to find simultaneous diophantine approximations.

Theorem 4. Let $N_{i}=p_{i} q_{i}, i=1 \ldots, k$, be $k \geq 3 n$-bit $R S A$ moduli where the $q_{i}$ 's are $\alpha$-bit primes. Suppose that there exist $k$ integers $a_{1}, \ldots, a_{k}$ with $a_{i} \leq 2^{\beta}$, $i=1, \ldots, k$, such that the $a_{i} p_{i}$ 's share all $t$ most significant bits. If

$$
t>\frac{k}{k-1} \alpha+\frac{k^{2}}{k-1} \beta+\frac{k}{2(k-1)}\left(1+\log _{2}(\pi e)\right),
$$

then, under the Gaussian Heuristic assumption, one can factor the $k$ RSA moduli $N_{1}, \cdots, N_{k}$ in polynomial time.

Proof. For $2 \leq i \leq k$, we set $x_{i}=a_{i} p_{i}-a_{1} p_{1}$. Then, multiplying by $q_{1} q_{i}$, we get $a_{i} q_{1} N_{i}-a_{1} q_{i} N_{1}=q_{1} q_{i} x_{i}$. Define $a=\prod_{j=1}^{k} a_{j}$. Multiplying by $\frac{a}{a_{i}}$, we get

$$
a q_{1} N_{i}-\frac{a a_{1} q_{i}}{a_{i}} N_{1}=\frac{a q_{1} q_{i} x_{i}}{a_{i}} .
$$

Let $C$ be a number to be fixed later. Consider the vector

$$
v=\left(C a q_{1}, \frac{a q_{1} q_{2} x_{2}}{a_{2}}, \ldots, \frac{a q_{1} q_{k} x_{k}}{a_{k}}\right) \in \mathbb{Z}^{k} .
$$


Then $v=\left(a q_{1}, \frac{a a_{1} q_{2}}{a_{2}} \ldots, \frac{a a_{1} q_{k}}{a_{k}}\right) \times M$, where $M$ is the $k \times k$-matrix

$$
M=\left[\begin{array}{cccccc}
C & N_{2} & N_{3} & \ldots & N_{k-1} & N_{k} \\
0 & -N_{1} & 0 & \ldots & 0 & 0 \\
0 & 0 & -N_{1} & \ldots & 0 & 0 \\
\vdots & \vdots & \vdots & \ddots & \vdots & \vdots \\
0 & 0 & 0 & \ldots & -N_{1} & 0 \\
0 & 0 & 0 & \ldots & 0 & -N_{1}
\end{array}\right] .
$$

Let $\mathcal{L}$ be the lattice defined by the rows of $M$. The dimension of $\mathcal{L}$ is $k$ and the determinant is $\operatorname{det}(\mathcal{L})=C N_{1}^{k-1}$. The Gaussian Heuristics for $\mathcal{L}$ asserts that the length of its shortest non-zero vector is usually $\sigma(\mathcal{L})$ where

$$
\sigma(\mathcal{L}) \approx \sqrt{\frac{k}{2 \pi e}} \operatorname{det}(\mathcal{L})^{\frac{1}{k}}=\sqrt{\frac{k}{2 \pi e}} C^{\frac{1}{k}} N_{1}^{\frac{k-1}{k}} .
$$

If we choose $C$ such that $\sigma(\mathcal{L})>\|v\|$, then $v$ can be found among the shortest non-zero vectors of the lattice $\mathcal{L}$. Using $(2)$, we get

$$
\|v\|^{2}=C^{2} a^{2} q_{1}^{2}+\sum_{i=2}^{k} \frac{a^{2} q_{1}^{2} q_{i}^{2} x_{i}^{2}}{a_{i}^{2}} .
$$

Suppose that for $i=1, \ldots, k$, we have

$$
N_{i} \approx 2^{n}, \quad q_{i} \approx 2^{\alpha}, \quad p_{i} \approx 2^{n-\alpha}, \quad a_{i} \leq 2^{\beta} .
$$

Moreover, suppose that the $a_{i} p_{i}$ 's share all $t$ MSBs. Then, for $i \geq 2$, we have

$$
\left|x_{i}\right|=\left|a_{i} p_{i}-a_{1} p_{1}\right| \leq 2^{n-\alpha+\beta-t} \text {. }
$$

Hence (4) leads to

$$
\begin{aligned}
\|v\|^{2} & <C^{2} \times 2^{2 k \beta+2 \alpha}+(k-1) 2^{2 k \beta+4 \alpha+2(n+\beta-\alpha-t)-2 \beta} \\
& =C^{2} \times 2^{2 k \beta+2 \alpha}+(k-1) \times 2^{2 k \beta+2 \alpha+2 n-2 t} .
\end{aligned}
$$

Define $C$ such that $C^{2} \times 2^{2 k \beta+2 \alpha} \geq 2^{2 k \beta+2 \alpha+2 n-2 t}$, that is $C \geq 2^{n-t}$. Then $\|v\|^{2}<k C^{2} \times 2^{2 k \beta+2 \alpha}$. On the other hand, using $N_{i} \approx 2^{n}$ in $(3)$, we get

$$
\sigma(\mathcal{L})^{2} \approx \frac{k}{2 \pi e} C^{\frac{2}{k}} \times 2^{\frac{2 n(k-1)}{k}} .
$$

Suppose $\sigma(L)>\|v\|$. Then $\sigma(L)^{2}>\|v\|^{2}$, that is

$$
\frac{k}{2 \pi e} C^{\frac{2}{k}} 2^{\frac{2 n(k-1)}{k}}>k C^{2} \times 2^{2 k \beta+2 \alpha} .
$$


Hence

$$
C^{\frac{2(k-1)}{k}}<\frac{1}{\pi e} 2^{\frac{2 n(k-1)}{k}-2 k \beta-2 \alpha-1} .
$$

Plugging $C \geq 2^{n-t}$ and extracting $t$, we get

$$
t>\frac{k}{k-1} \alpha+\frac{k^{2}}{k-1} \beta+\frac{k}{2(k-1)}\left(1+\log _{2}(\pi e)\right) .
$$

Using (2), we get $q_{1}=\operatorname{gcd}\left(C a q_{1}, N_{1}\right)$ and for $i=2, \ldots, k, q_{i}=\operatorname{gcd}\left(\frac{a a_{1} q_{i}}{a_{i}}, N_{i}\right)$. This terminates the proof.

We notice that with $\beta=0$, that is $a_{i}=1$ for $i=1, \ldots, k$, we get

$$
t>\frac{k}{k-1} \alpha+\frac{k}{2(k-1)}\left(1+\log _{2}(\pi e)\right)
$$

which slightly improves the bound obtained by Faugère et al. in [1. This shows that our result extends the result of Faugère et al. where they considered only the case when the $p_{i}$ 's share $t$ MSBs.

\section{Factoring Two RSA Moduli in the LSB Case}

The study of implicit factorization when $p_{1}, p_{2}$ share some LSBs has been considered in [5], 8], [1] and [3]. In this section, we extend the former attacks to the case where an unknown multiple $a_{1} p_{1}$ of $p_{1}$ and an unknown multiple $a_{2} p_{2}$ of $p_{2}$ share their $t$ LSBs.

\subsection{The general attack}

Theorem 5. Let $N_{1}=p_{1} q_{1}, N_{2}=p_{2} q_{2}$ be two RSA moduli. Assume that there exist two integers $a_{1}, a_{2}$ with $a_{1}<p_{2}, a_{2}<p_{1}$ such that $a_{1} p_{1}$ and $a_{2} p_{2}$ share $t$ many LSBs. If $a_{1} a_{2} q_{1} q_{2}<2^{t-1}$, then one can factor $N_{1}$ and $N_{2}$ in polynomial time.

Proof. Let $N_{1}=p_{1} q_{1}$ and $N_{2}=p_{2} q_{2}$. Assume that $a_{1} p_{1}$ and $a_{2} p_{2}$ share $t$ many LSBs. Then $a_{1} p_{1}-a_{2} p_{2}=2^{t} x$ for some integer $x$ and we have

$$
q_{1} q_{2}\left(a_{1} p_{1}-a_{2} p_{2}\right)=N_{1} a_{1} q_{2}-N_{2} a_{2} q_{1}=2^{t} x q_{1} q_{2} .
$$

Then $N_{1} a_{1} q_{2}-N_{2} a_{2} q_{1} \equiv 0\left(\bmod 2^{t}\right)$. Since $\operatorname{gcd}\left(N_{1}, 2\right)=1$, then $N_{1}^{-1}\left(\bmod 2^{t}\right)$ exists and $a_{1} q_{2}-a_{2} q_{1} N_{2} N_{1}^{-1} \equiv 0\left(\bmod 2^{t}\right)$. Define $T \equiv N_{2} N_{1}^{-1}\left(\bmod 2^{t}\right)$. Then $a_{1} q_{2}-a_{2} q_{1} T \equiv 0\left(\bmod 2^{t}\right)$ and there exists an integer $y$ such that

$$
a_{1} q_{2}=a_{2} q_{1} T-2^{t} y .
$$

Suppose that $a_{1} a_{2} q_{1} q_{2}<2^{t-1}$. Then dividing by $2^{t} a_{2} q_{1}$, we get

$$
\left|\frac{T}{2^{t}}-\frac{y}{a_{2} q_{1}}\right|=\frac{\left|a_{2} q_{1} T-2^{t} y\right|}{2^{t} a_{2} q_{1}}=\frac{a_{1} q_{2}}{2^{t} a_{2} q_{1}}<\frac{a_{1} q_{2}}{2 a_{1} a_{2} q_{1} q_{2} a_{2} q_{1}}=\frac{1}{2\left(a_{2} q_{1}\right)^{2}} .
$$


Therefore from Theorem 1 it follows that $\frac{y}{a_{2} q_{1}}$ is one of the convergents in the continued fraction expansion of $\frac{T}{2^{t}}$. Since $a_{2}<p_{1}$, we get $q_{1}=\operatorname{gcd}\left(N 1, a_{2} q_{1}\right)$ and $p_{1}=\frac{N_{1}}{q_{1}}$. Using (5), we get $a_{1} q_{2}=a_{2} q_{1} T-2^{t} y$. Similarly, since $a_{1}<p_{2}$, we get $q_{2}=\operatorname{gcd}\left(N_{2}, a_{1} q_{2}\right)$ and $p_{2}=\frac{N_{2}}{q_{2}}$. This terminates the proof.

\subsection{Application to unbalanced RSA and RSA for Paranoids}

Here we apply Theorem 5 in the situation that the two RSA moduli $N_{1}=p_{1} q_{1}$, $N_{2}=p_{2} q_{2}$ are of the same shape, that is $N_{1}$ and $N_{2}$ are of the same bit-size and the $q_{i}$ 's are $\alpha$-bit primes.

Corollary 2. Let $N_{1}=p_{1} q_{1}, N_{2}=p_{2} q_{2}$ be two unbalanced $n$-bit size $R S A$ moduli with $q_{1} \approx q_{2} \approx 2^{\alpha}$. Suppose that there exist two positive integers $a_{1} \leq 2^{\beta}$, $a_{2} \leq 2^{\beta}$ such that $a_{1} p_{1}$ and $a_{2} p_{2}$ share the $t$ LSBs. If $t \geq 2 \alpha+2 \beta+1$, then one can factor $N_{1}$ and $N_{2}$ in polynomial time.

Proof. Let $N_{1}=p_{1} q_{1}, N_{2}=p_{2} q_{2}$ be two RSA moduli with $N_{1} \approx N_{2} \approx 2^{n}$ and, $q_{1} \approx q_{2} \approx 2^{\alpha}$. Suppose that a multiple $a_{1} p_{1}$ and a multiple $a_{2} p_{2}$ share the $t$ least significant bits where $a_{i} \leq 2^{\beta}$ for $i=1,2$. Define $T \equiv N_{2} N_{1}^{-1}\left(\bmod 2^{t}\right)$. As in the proof of Theorem 5, we have $a_{1} p_{1}-a_{2} p_{2}=2^{t} x$ and $a_{1} q_{2}=a_{2} q_{1} T-2^{t} y$ for some integers $x$ and $y$. Suppose that $t \geq 2 \alpha+2 \beta+1$. Then $a_{1} a_{2} q_{1} q_{2}<2^{2 \beta+2 \alpha} \leq 2^{t-1}$. Therefore, using the same arguments than Theorem 5. we conclude that $\frac{y}{a_{2} q_{1}}$ is one of the convergents in the continued fraction expansion of $\frac{T}{2^{t}}$ which leads to the factorization of $N_{1}$ and $N_{2}$.

Remark 3. Here again, if $\beta=0$, then the condition of Corollary 2 becomes $t \geq$ $2 \alpha+1$ which improves the bounds found in the former approaches of [5], 8], [1] and retrieves the bound of $[3]$.

As an application of Corollary 2 , consider two 1024-bit RSA for Paranoids moduli $N_{1}=p_{1} q_{1}, N_{2}=p_{2} q_{2}$ where $q_{1}, q_{2}$ are 500-bit primes. Hence $\alpha=500$ and using Corollary 2. one can factor $N_{1}$ and $N_{2}$ if there exist two integers $a_{1} \leq 2^{\beta}$ and $a_{2} \leq 2^{\beta}$ such that $a_{1} p_{1}$ and $a_{2} p_{2}$ share $t$ LSBs with $t>2001+2 \beta$.

\section{Factoring $k$ RSA Moduli in the LSB Case}

In this section, we assume that we are given $k \geq 3$ different RSA moduli $N_{i}=$ $p_{i} q_{i}, i=1, \ldots, k$ where some unknown multiples $a_{i} p_{i}$ 's coincide on the $t$ least significant bits. For suitably large $t$, we show that there is an efficient algorithm that recovers the factorization of the $k$ RSA moduli. To this end, we use the lattice reduction techniques to solve a simultaneous diophantine approximations problem.

Theorem 6. Let $N_{i}=p_{i} q_{i}, i=1 \ldots, k$, be $k \geq 3 n$-bit RSA moduli where the $q_{i}$ 's are $\alpha$-bit primes. Suppose that there exist $k$ positive integers $a_{1}, \ldots, a_{k}$ with $a_{i} \leq 2^{\beta}, i=1, \ldots, k$, such that the $a_{i} p_{i}$ 's share all $t$ least significant bits. If

$$
t>\frac{k}{k-1} \alpha+\frac{k^{2}}{k-1} \beta+\frac{k}{2(k-1)}\left(1+\log _{2}(\pi e)\right),
$$


then, under the Gaussian Heuristic assumption, one can factor the $k$ RSA moduli $N_{1}, \cdots N_{k}$ in polynomial time.

Proof. For $1 \leq i \leq k$, suppose that the $a_{i} p_{i}$ 's share $t$ least significant bits. Then, for $1 \leq i \leq k, a_{i} p_{i}-a_{1} p_{1}=2^{t} x_{i}$. Multiplying by $q_{1} q_{i}$, we get $a_{i} q_{1} N_{i}-a_{1} q_{i} N_{1}=$ $2^{t} q_{1} q_{i} x_{i}$. Define $a=\prod_{j=1}^{k} a_{j}$. Multiplying by $\frac{a}{a_{i}}$, we get

$$
a q_{1} N_{i}-\frac{a a_{1} q_{i}}{a_{i}} N_{1}=\frac{2^{t} a q_{1} q_{i} x_{i}}{a_{i}} .
$$

Transforming modulo $2^{t}$, we get $a q_{1} N_{i} N_{1}^{-t}-\frac{a a_{1} q_{i}}{a_{i}} \equiv 0\left(\bmod 2^{t}\right)$. Define $T_{i} \equiv$ $N_{i} N_{1}^{-1}\left(\bmod 2^{t}\right)$. Then $a q_{1} T_{i}-\frac{a a_{1} q_{i}}{a_{i}} \equiv 0\left(\bmod 2^{t}\right)$ and there exists an integer $y_{i}$ such that $a q_{1} T_{i}-2^{t} y_{i}=\frac{a a_{1} q_{i}}{a_{i}}$. Consider the vector

$$
v=\left(a q_{1}, \frac{a a_{1} q_{2}}{a_{2}}, \ldots, \frac{a a_{1} q_{k}}{a_{k}}\right) \in \mathbb{Z}^{k} .
$$

Then $v=\left(a q_{1}, y_{2} \ldots, y_{k}\right) \times M$, where $M$ is the $k \times k$-matrix

$$
M=\left[\begin{array}{cccccc}
1 & T_{2} & T_{3} & \ldots & T_{k-1} & T_{k} \\
0 & -2^{t} & 0 & \ldots & 0 & 0 \\
0 & 0 & -2^{t} & \ldots & 0 & 0 \\
\vdots & \vdots & \vdots & \ddots & \vdots & \vdots \\
0 & 0 & 0 & \ldots & -2^{t} & 0 \\
0 & 0 & 0 & \ldots & 0 & -2^{t}
\end{array}\right] .
$$

Let $\mathcal{L}$ be the lattice defined by the rows of the matrix $M$. The dimension of $\mathcal{L}$ is $k$ and the determinant is $\operatorname{det}(\mathcal{L})=2^{(k-1) t}$. The Gaussian Heuristics for $\mathcal{L}$ asserts that the length of its shortest non-zero vector is $\sigma(\mathcal{L})$ where

$$
\sigma(\mathcal{L}) \approx \sqrt{\frac{k}{2 \pi e}} \operatorname{det}(\mathcal{L})^{\frac{1}{k}}=\sqrt{\frac{k}{2 \pi e}} 2^{\frac{(k-1) t}{k}} .
$$

Observe that the norm of $v$ satisfies

$$
\|v\|^{2}=a^{2} q_{1}^{2}+\sum_{i=2}^{k}\left(\frac{a a_{1} q_{k}}{a_{k}}\right)^{2} .
$$

If the $a_{i} p_{i}$ 's share all $t$ least significant bits, then, for $i=1, \ldots, k$, we have

$$
q_{i} \approx 2^{\alpha}, \quad a_{i} \leq 2^{\beta}, \quad\left|x_{i}\right|=\frac{\left|a_{i} p_{i}-a_{1} p_{1}\right|}{2^{t}}<2^{n-\alpha+\beta-t} .
$$

Hence

$$
\|v\|^{2}<2^{2 k \beta+2 \alpha}+(k-1) 2^{2 k \beta+2 \alpha}=k 2^{2 k \beta+2 \alpha} .
$$


Using (8) and 77) and transforming $\sigma(L)^{2}>\|v\|^{2}$ into $\frac{k}{2 \pi e} 2^{\frac{2(k-1) t}{k}}>k 2^{2 k \beta+2 \alpha}$, we get

$$
t>\frac{k}{k-1} \alpha+\frac{k^{2}}{k-1} \beta+\frac{k}{2(k-1)}\left(1+\log _{2}(\pi e)\right) .
$$

Using (6), we get $q_{1}=\operatorname{gcd}\left(a q_{1}, N_{1}\right)$ and for $i=2, \ldots, k, q_{i}=\operatorname{gcd}\left(\frac{a a_{1} q_{i}}{a_{i}}, N_{i}\right)$. This terminates the proof.

Once again, if $\beta=0$, then $a_{i}=1$ and the bound of Theorem 6 transforms to $t>\frac{k}{k-1} \alpha+\frac{k}{2(k-1)}\left(1+\log _{2}(\pi e)\right)$, which improves the bound of [1].

\section{Experiments}

In this section, we describe the experiments that we conducted for $k=4,10,30$ and 50 RSA moduli, in connection with Theorem 4 and Theorem 6 . We verified our assumptions by running experiments on a Core 2 Duo $2 \mathrm{GHz}$ notebook. The lattice reduction basis technique was based on the LLL algorithm.

Assume that $a_{1} p_{1}$ and the $a_{i} p_{i}$ 's share $t$ MSBs. Then since $a_{i} p_{i} \leq 2^{n-\alpha+\beta}$, we see that $\left|a_{i} p_{i}-a_{1} p_{1}\right| \leq 2^{n-\alpha+\beta-t}$. Therefore, $t \leq n-\alpha+\beta$. Similarly, assume that $a_{1} p_{1}$ and the $a_{i} p_{i}$ 's share $t$ LSBs. Then $\left|a_{i} p_{i}-a_{1} p_{1}\right|=2^{t} x_{i}$ with $t \leq n-\alpha+\beta$. In both cases, combining with the bound of $t$ in Theorem 4 and Theorem 5 , we get

$$
n-\alpha+\beta \geq t>\frac{k}{k-1} \alpha+\frac{k^{2}}{k-1} \beta+\frac{k}{2(k-1)}\left(1+\log _{2}(\pi e)\right),
$$

which is satisfied if

$$
\beta<\frac{n(k-1)}{k^{2}-k+1}-\frac{2 k-1}{k^{2}-k+1} \alpha-\frac{k}{2\left(k^{2}-k+1\right)}\left(1+\log _{2}(\pi e)\right) .
$$

Consequently, we only consider the situation where the bit-size $\beta$ of the $a_{i}$ 's satisfies condition (9).

We generated many random 1024-bit RSA moduli for $k=4,10,30,50$ and various values of $\alpha$ and $\beta$ according to the bound (9). All our experiments were successful and the assumptions on the Gaussian Heuristics were verified. In Table 4 we notice the experimentally lowest values of $t$ that have $100 \%$ success rate.

\section{Conclusion}

In this work we have designed a technique to factor $k \geq 2$ RSA moduli $N_{i}=p_{i} q_{i}$, $i=1, \ldots, k$ when some unknown multiples $a_{i} p_{i}$ share $t$ many Most Significant Bits (MSBs) or $t$ many Least Significant Bits (LSBs). The new technique generalizes many previous results where the prime factors $p_{i}$ share $t$ many MSBs or $t$ many LSBs. This provides practitioners tighter conditions for the primes 
Table 4. Experiments for $k$ RSA moduli in the MSB and the LSB cases.

\begin{tabular}{|c|c|c|c|c|c|c|c|}
\hline \begin{tabular}{|c|} 
Number \\
$k$ \\
of \\
moduli \\
\end{tabular} & $\begin{array}{c}\text { Bit-size } \\
\alpha \text { of } \\
\text { the } \\
q_{i} \text { 's }\end{array}$ & $\begin{array}{c}\text { Max } \\
\text { bit-size } \\
\beta \text { of the } \\
a_{i} \text { 's } 9\end{array}$ & $\begin{array}{c}\text { Used } \\
\text { bit-size } \\
\beta \text { of } \\
\text { the } a_{i} \text { 's }\end{array}$ & $\begin{array}{c}\text { Minimal } \\
\text { theoretical } \\
\text { bound } \\
\text { for } t\end{array}$ & $\begin{array}{c}\text { Experimental } \\
\text { bound } \\
\text { for } t \text { in } \\
\text { MSB case }\end{array}$ & $\begin{array}{c}\text { Experimental } \\
\text { bound } \\
\text { for } t \text { in } \\
\text { LSB case }\end{array}$ & $\begin{array}{l}\text { Number } \\
\text { of } \\
\text { experi- } \\
\text { ments }\end{array}$ \\
\hline 4 & 150 & 154 & 100 & 737 & 602 & 611 & 1000 \\
\hline 4 & 250 & 100 & 80 & 763 & 655 & 662 & 1000 \\
\hline 4 & 350 & 46 & 35 & 657 & 609 & 616 & 1000 \\
\hline 4 & 400 & 20 & 15 & 617 & 594 & 601 & 1000 \\
\hline 10 & 150 & 69 & 50 & 725 & 649 & 674 & 1000 \\
\hline 10 & 250 & 48 & 40 & 725 & 667 & 684 & 1000 \\
\hline 10 & 350 & 27 & 20 & 614 & 591 & 603 & 1000 \\
\hline 10 & 400 & 17 & 12 & 581 & 563 & 570 & 1000 \\
\hline 30 & 150 & 23 & 15 & 623 & 585 & 592 & 500 \\
\hline 30 & 250 & 17 & 12 & 634 & 596 & 603 & 500 \\
\hline 30 & 350 & 10 & 8 & 613 & 544 & 572 & 500 \\
\hline 30 & 400 & 6 & 4 & 541 & 533 & 536 & 500 \\
\hline 50 & 150 & 14 & 10 & 666 & 648 & 650 & 100 \\
\hline 50 & 250 & 10 & 7 & 615 & 597 & 605 & 100 \\
\hline 50 & 350 & 6 & 4 & 564 & 546 & 551 & 100 \\
\hline 50 & 400 & 4 & 3 & 564 & 556 & 559 & 100 \\
\hline
\end{tabular}

that are generated for utilization with the RSA algorithm. On the other hand, our results also serve their purpose to provide a peace of mind for practitioners knowing that the generated RSA moduli does not fall into any of the categories mentioned in this work.

\section{References}

1. Faugère, J-C., Marinier, R., Renault, G.: Implicit factoring with shared most significant and middle bits. In P.Q. Nguyen and D. Pointcheval (Eds.): Public Key Cryptography, Lecture Notes in Computer Science, Springer 6056 (2010) 70-87

2. Hardy, G.H., Wright, E.M.: An Introduction to the Theory of Numbers. Oxford University Press, London (1975)

3. Kurosawa, K., Ueda, T.: How to factor $N_{1}$ and $N_{2}$ when $p_{1}=p_{2}\left(\bmod 2^{t}\right)$. In K. Sakiyama and M. Terada (Eds.): IWSEC 2013, Lecture Notes in Computer Science, Springer 8231 (2013) 217-225

4. Lenstra, A.K., Lenstra, H.W., Lovász., L.: Factoring polynomials with rational coefficients. Mathematische Annalen 261 (1982) 513-534

5. May, A., M. Ritzenhofen, R.: Implicit factoring: On polynomial time factoring given only an implicit hint. In Stanislaw Jarecki and Gene Tsudik (Eds.): Public Key Cryptography, Lecture Notes in Computer Science, Springer 5443 (2009) 1-14

6. Rivest,R., Shamir, A., Adleman, L.: A method for obtaining digital signatures and public-key cryptosystems. Communications of the ACM 21 (1978) 120-126

7. Shamir, A: RSA for Paranoids. RSA Laboratories CryptoBytes 1 (1995) 3-4 
8. Sarkar, S., Maitra.,S.: Further results on implicit factoring in polynomial time. Advances in Mathematics of Communications 3 (2009) 205-217 